\title{
Histopathology is ripe for automation
}

\author{
Advances in microscopy and the application of machine learning to histology will modernize the examination of \\ tissues in the clinical laboratory and in the operating room.
}

N edical devices and technology available in hospitals and medical laboratories in the developed world have dramatically improved healthcare. Computed tomography scanners, realtime ultrasonography, rapid HIV tests, laparoscopic surgery and electronic health records are notable examples. In contrast, clinical histopathology - which involves tissue-processing steps such as fixation, embedding in paraffin wax, sectioning, slide mounting, staining with dyes, and subsequent microscopy analysis of the tissue slides to identify histopathological features - has essentially remained unchanged for decades.

For many diseases, histopathology is the clinical gold-standard technique used for diagnosis, and in research laboratories it is typically used to benchmark or confirm findings from basic and translational biomedical research. Because tissue processing is a laborious process that can take hours and sometimes days, the typical amount of tissue that can be processed from a biopsy represents only a small fraction of the whole sample, which limits diagnostic assessment to a few regions of interest that may not be representative of disease. Also, the handling of biopsied tissue samples requires high levels of manual dexterity at each step of the process and can result in artefacts, which can, for example, originate from squashed or torn sections, and from inconsistencies in staining (which can be caused by variations in the strength of the crosslinking effect of formalin, a chemical fixative). Soon the convergence of advances in microscopy, digital-image processing and machine learning could eliminate most of the tissue-handling steps, and enable rapid diagnostic histopathology of entire biopsied tissues in the laboratory. It would also enable large sections of fresh tissue to be assessed in the operating room with unprecedented resolution, and with contrast and diagnostic accuracy rivalling that of expert pathologists.

To this end, Richard Levenson and colleagues describe in this issue a simple and fast histology approach that eliminates the need for tissue mounting on microscopy slides. The method involves soaking whole tissue samples with fluorescent dyes, whose surface emission under ultraviolet illumination can then be visualized within minutes through a camera with standard

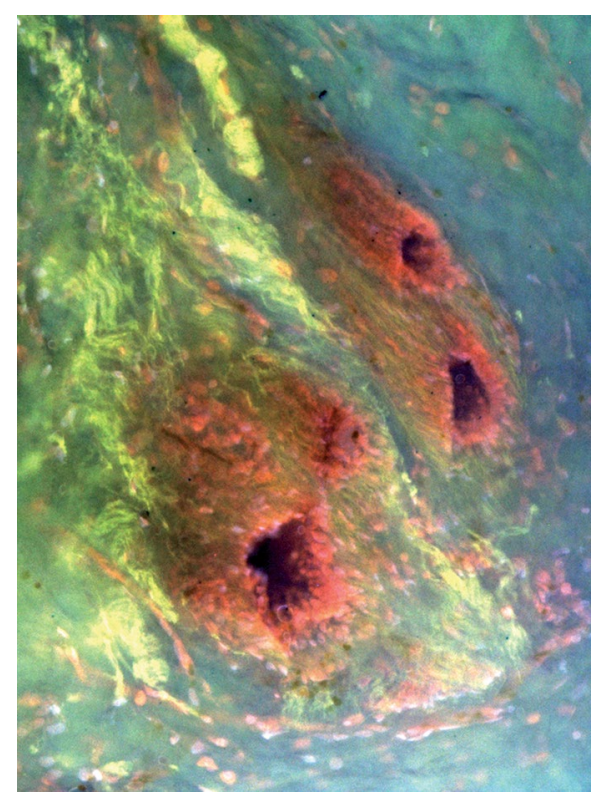

Credit: Richard Levenson, University of California Davis

optics. The images (such as that pictured) can then be modified with image-processing software to create virtual haematoxylin and eosin renderings. Fluorescent images and their virtual renderings were used in a blinded pilot study involving a range of tissue types that led individual pathologists to reach diagnoses that were in agreement with those obtained through conventional histopathology. The approach is compatible with samples obtained from a wide variety of cancer types and can also provide topographical information (because ultraviolet light has a larger penetration depth than white light).

Stained tissue samples can also be imaged through a modified light-sheet fluorescence microscope, as recently reported by Jonathan Liu and colleagues (Nat. Biomed. Eng. 1, 0084; 2017). The approach optically 'sections' the sample, hence enabling volumetric imaging by stacking virtual slices (each taking less than 10 minutes to image). Optical sectioning can be coupled with optical clearing techniques - which use liquid solutions that make tissue optically transparent while retaining its three-dimensional structure - that allow for further staining. Although clearing protocols lengthen tissue-processing times, they enable the volumetric imaging of microstructures in large, fixed samples, and can be combined with antibody staining for the assessment of protein expression. Using such an approach, Per Uhlén and co-authors (Nat. Biomed. Eng. $1,796-806$; 2017) recently demonstrated that the intratumoural heterogeneity of fixed, embedded and cleared tumour biopsies, including samples that had been stored for a long time, can be rapidly visualized via light-sheet microscopy. They also showed that three-dimensional characterization of tumour microvessels in samples from patients with ovarian cancers enabled the stratification of their prognosis.

Tissue staining can be circumvented by using spectroscopic techniques that take advantage of endogenous contrast generated from tissue components. As shown earlier this year by Daniel Orringer and co-authors (Nat. Biomed. Eng. 1, 0027; 2017), stimulated Raman scattering microscopy (a label-free optical technique) can generate histology images that, after having been processed with software to create virtual haematoxylin and eosin colouring, could be used by neuropathologists to identify brain-tumour subtypes in a set of clinical tissue samples as accurately as conventional histology images. The authors also showed that a machine-learning algorithm trained with a different set of samples diagnosed the same test set with $90 \%$ accuracy.

Rapid and automated histology approaches that offer three-dimensional views of molecular biomarkers and cytological features in unprocessed or minimally processed tissue, and that enable the quantitative annotation of histological images and their segmentation by intelligent algorithms that 'learn' the physical patterns of disease, will increase diagnostic accuracy and facilitate disease classification and decision-making in the clinic. However, the diffusion of these technologies into clinical laboratories and hospitals will take years, and will require the adaptation of the techniques and protocols to clinical workflows, the development, testing and deployment of cost-effective implementations, and the training of laboratory personnel and clinicians. For histopathology, the twentyfirst century can't come soon enough.

Published online: 12 December 2017 https://doi.org/10.1038/s41551-017-0179-5 